\title{
DIFFERENCE METHODS AND DEFERRED CORRECTIONS FOR ORDINARY BOUNDARY VALUE PROBLEMS*
}

\author{
H. B. KELLER $\dagger$ AND V. PEREYRA $\dagger$
}

\begin{abstract}
Compact as possible difference schemes for systems of $n$th order equations are developed. Generalizations of the Mehrstellenverfahren and simple theoretically sound implementations of deferred corrections are given. It is shown that higher order systems are more efficiently solved as given rather than as reduced to larger lower order systems. Tables of coefficients to implement these methods are included and have been derived using symbolic computations.
\end{abstract}

1. Introduction. High order accurate numerical methods seem to be most efficient for solving general classes of two point boundary value problems. In particular Richardson extrapolation and deferred corrections applied to "low" order accurate finite difference schemes are most effective. The former procedure has been theoretically justified for first order systems [5], [6] and it has been implemented using the trapezoidal rule [13]. Deferred corrections seem to be even more efficient and have been implemented for first order systems [13], [21]. However the codes are more complicated and some slight gaps remain in the theory [15], [17]. In this paper we seek to eliminate these defects and to devise methods for treating systems of any order. We have also implemented some of these methods for even order systems.

Specifically we consider first general linear $n$th order systems of the form:

$$
\mathscr{L} y(t) \equiv \sum_{\nu=0}^{n} A_{\nu}(t) \mathscr{D}^{\nu} y(t)=f(t), \quad a \leqq t \leqq b .
$$

Here $\mathscr{D} \equiv d / d t, y(t)$ and $f(t)$ are $d$-vectors, $A_{\nu}(t)$ are $d \times d$ matrices with $A_{n}(t)$ nonsingular on $[a, b]$ or frequently $A_{n}(t) \equiv I$. All functions are in $C^{M}[a, b]$ for some sufficiently large $M$. The $n d$ linearly independent boundary conditions are

$$
B_{k} y \equiv \sum_{\nu=0}^{n-1}\left[B_{k \nu}(a) \mathscr{D}^{\nu} y(a)+B_{k \nu}(b) \mathscr{D}^{\nu} y(b)\right] \equiv g_{k}, \quad 0 \leqq k \leqq n-1 .
$$

Here the matrices $B_{\nu k}(x), x=a, b$ are $d \times d$ and with them we define the $d n \times d n$ matrices

$$
B(a) \equiv\left(B_{k \nu}(a)\right), \quad B(b) \equiv\left(B_{k \nu}(b)\right)
$$

which are required to satisfy:

$$
\operatorname{rank}(B(a), B(b))=d n \text {. }
$$

Thus there are $d n$ independent constraints on the solution and we assume that the problem (1.1), (1.2) has a unique smooth solution, $y(t) \in C^{M+n}[a, b]$. Equivalently the homogeneous problem, with $f(t) \equiv 0, g_{k} \equiv 0,0 \leqq k \leqq n-1$, has only the trivial solution.

Under the above conditions H.-O. Kreiss [12] has developed a very general theory of finite difference methods for problems of the form (1.1), (1.2) (but with the $B(a)$ and $B(b)$ block lower triangular.) We shall employ only schemes which are "compact as possible" and "centered". For simplicity we call them centered compact schemes. One

* Received by the editors August 2, 1977. This work was supported by the Energy Research and Development Administration under Contract AT-04-3-767, Project Agreement 12.

† Department of Applied Mathematics, California Institute of Technology, Pasadena, California 91125. 
trivial extension of the theory is required. We note that the functions $A_{\nu}(t), 0 \leqq \nu \leqq n$ and $f(t)$ can be extended smoothly, say to $C^{M}[a-\delta, b+\delta]$ for some fixed but (arbitrarily) small $\delta>0$. This is assured, for example, by the Tietze extension theorem. Using these extensions and the uniqueness theorem for initial value problems for (1.1) the solution $y(t)$ of (1.1), (1.2) can be uniquely extended, as a solution of (1.1), to $C^{M+n}[a-\delta, b+\delta]$. Our centered compact schemes will be employed at all netpoints on $[a, b]$. These difference equations and the boundary approximations may use netpoints which spill over into $[a-\delta, b+\delta]$. The Kreiss theory remains unaltered by these trivial but crucial extensions.

In $\S 2$ we formulate centered compact schemes and show that they are second order accurate with truncation expansions which proceed in powers of $h^{2}$. A generalization of the Mehrstellenverfahren is derived in $\S 2.1$ where centered compact schemes of accuracy $2 p$ are derived provided $A_{n-1}(t) \equiv A_{n-2}(t) \equiv \cdots \equiv A_{n-2 p+3}(t) \equiv 0$. The $h^{2}$ truncation expansion remains valid for this scheme. An extension to semi-linear problems is presented in $\S 3$. The general deferred correction theory is sketched in $\S 4$ and two methods for circumventing the end of the net catastrophy are sketched in $\S 5$. Some details of the theoretically most sound of these precedures are given in $\S 5.1$. An implementation of the entire scheme including deferred corrections is described in $\S 6$. Finally in $\S 7$ we show some theoretical and practical advantages of retaining high order systems as given rather than reducing them to lower order but larger systems.

The basic idea of many of the techniques used in this paper can be traced back to Fox [3]. Proofs and complete detailed formulas are not always presented in [3] but deferred corrections for higher order schemes is stressed and the use of netpoints outside the basic interval $[a, b]$ is also employed. More recently we have learned of a report by Cerutti [22] in which collocation methods for general higher order systems are discussed in some detail.

2. Centered compact schemes. On the interval $[a-\delta, b+\delta]$ we introduce two sequences of uniform nets, $\pi_{h}^{\theta}, \theta=e$ and $\theta=o$, with net points $\left\{t_{j}\right\}$ defined by

$$
t_{j}=t_{0}^{\theta}+j h, \quad h=\frac{b-a}{J}, \quad j=-r,-r+1, \cdots, J+r .
$$

We use $t_{0}^{o} \equiv a$ in defining $\pi_{h}^{o}$ and $t_{0}^{e} \equiv a-h / 2$ in defining $\pi_{h}^{e}$. When $n=2 m+1$ is odd we seek approximations $u^{h} \equiv\left\{u_{j}\right\}$ to $\left\{y\left(t_{j}\right)\right\}$ on the odd net, $\pi_{h}^{o}$, while for $n=2 m$ the approximations, $u^{h}$, are sought on $\pi_{h}^{e}$, the even net. (Note that the even net is simply the odd net shifted $h / 2$ units to the left.) The integer $r$ will be fixed, independently of $h$, in terms of $n$ and the ultimate order of accuracy desired. For the present we need only require that $r h \leqq \delta$. The difference schemes we study employ as few adjacent netpoints as possible to approximate $\mathscr{D}^{n} y(t)$ in the differential equations and to approximate $\mathscr{D}^{n-1} y(a)$ and $\mathscr{D}^{n-1} y(b)$ in the boundary conditions. Thus they use $n+1$ netpoints in each difference pattern in approximating the differential equations (1.1) and $n$ netpoints near each endpoint in approximating the boundary conditions (1.2). The accuracy thus obtained is at least $\mathscr{O}\left(h^{2}\right)$ as we shall see. These difference schemes have the forms:

$$
\mathscr{L}^{h} u_{j} \equiv \sum_{s=-\bar{m}}^{m} C_{s}\left(t_{j}, h\right) u_{j+s}=f^{h}\left(t_{j}\right), \quad 1 \leqq j \leqq J
$$

b) $\mathscr{B}_{k}^{h} u^{h} \equiv \sum_{s=-m}^{m}\left[C_{k s}(a, h) u_{s}+C_{k s}(b, h) u_{J+s}\right]=g_{k}^{h}, \quad 0 \leqq k \leqq n-1$. 
Here we use the integers $\bar{m}$ and $\underline{m}$ as given below.

$$
\bar{m} \equiv\left\{\begin{array}{c}
m+1 \\
m
\end{array}, \quad \underline{m} \equiv\left\{\begin{array} { c } 
{ m } \\
{ m - 1 }
\end{array} \text { if } \quad n \equiv \left\{\begin{array}{c}
2 m+1 \\
2 m
\end{array}, \quad \boldsymbol{\theta} \equiv\left\{\begin{array}{l}
o \\
e
\end{array} .\right.\right.\right.\right.
$$

The $d \times d$ matrices $C_{s}\left(t_{j}, h\right)$ and $C_{k s}(x, h)$ for $x=a$ or $b$ are polynomials in $h^{-1}$ while $f^{h}\left(t_{j}\right)$ and $g_{k}^{h}$ are approximations to $f(t)$ and $g_{k}$. It easily follows, since each $u_{j} \in \mathbb{E}^{d}$, that $(2.2 \mathrm{a}, \mathrm{b})$ represents $d(J+n)$ linear equations in as many unknowns.

As usual the truncation errors for (2.2a) are defined, for all sufficiently smooth solutions $y(t)$ of $(1.1)$, as

$$
\text { a) } \tau_{j}[y] \equiv \mathscr{L}^{h} y\left(t_{j}\right)-f^{h}\left(t_{j}\right), \quad 1 \leqq j \leqq J .
$$

Similarly the truncation errors in (2.2b) are defined, for all sufficiently smooth solutions $y(t)$ of $(1.2)$, using $y^{h} \equiv\left\{y\left(t_{j}\right)\right\}$, as

$$
\text { b) } \sigma_{k}[y] \equiv \mathscr{B}_{k}^{h} y^{h}-g_{k}^{h}, \quad 0 \leqq k \leqq n-1 .
$$

We say that $(2.2 \mathrm{a}, \mathrm{b})$ is consistent (or accurate) of order $p$ for (1.1)-(1.2) if for all smooth solutions, $y(t)$ of $(1.1)-(1.2)$ :

$$
\left\|\tau_{j}[y]\right\|=\mathscr{O}\left(h^{p}\right), \quad\left\|\sigma_{k}[y]\right\|=\mathscr{O}\left(h^{p}\right) .
$$

The scheme $\left\{\mathscr{L}^{h}, \mathscr{B}_{k}^{h}\right\}$ defined in $(2.2 \mathrm{a}, \mathrm{b})$ is stable provided there are positive constants $K, h_{0}$ such that for all net functions $\left\{v_{j}\right\}$ on all nets with $h \leqq h_{0}$ :

$$
\left\|v_{i}\right\| \leqq K\left\{\max _{1 \leqq j \leqq J}\left\|\mathscr{L}^{h} v_{j}\right\|+\max _{0 \leqq k<n}\left\|\mathscr{B}_{k}^{h} v^{h}\right\|\right\}, \quad-\underline{m} \leqq i \leqq J+m .
$$

The basic result of Kreiss [12] can now be formulated in our terminology, assuming $m h_{0}<\delta$, as follows.

THEOREM (2.7) (Kreiss). Let (1.1)-(1.2) have a unique solution $y(t)$ on $[a-\delta, b+$ $\delta$ ]. Let the compact scheme (2.2) be consistent for (1.1)-(1.2). Then $\left\{\mathscr{L}^{h}, \mathscr{B}_{k}^{h}\right\}$ of $(2.2 \mathrm{a}, \mathrm{b})$ is stable. For each $h \leqq h_{0}$ the unique solution $u^{h}$ of (2.2) satisfies:

$$
\left\|y\left(t_{i}\right)-u_{i}\right\| \leqq K\left\{\max _{1 \leqq j \leqq J}\left\|\tau_{j}[y]\right\|+\max _{0 \leqq k<n}\left\|\sigma_{k}[y]\right\|\right\}, \quad-\underline{m} \leqq i \leqq J+m .
$$

The boundary approximations, $(2.2 \mathrm{~b})$, need not be compact in the Kreiss theory. But for our study of practical high order accurate schemes we shall always make them compact. This reduces some of the constants in the error expansions and makes it easier to automate our procedures.

To write down centered compact schemes we use the standard difference and averaging operators:

$$
\begin{array}{lll}
D_{+} v_{j} \equiv h^{-1}\left(v_{j+1}-v_{j}\right), & D_{-} v_{j} \equiv h^{-1}\left(v_{j}-v_{j-1}\right), \\
D_{0} v_{j} \equiv(2 h)^{-1}\left(v_{j+1}-v_{j-1}\right), & M_{ \pm} v_{j} \equiv 2^{-1}\left(v_{j \pm 1}+v_{j}\right) .
\end{array}
$$

For even $n=2 m$ we use $u^{h}$ on the net $\pi_{h}^{e}$ and approximate (1.1) at each point in $[a, b] \cap \pi_{h}^{e}$ by:

$$
\text { a) } \mathscr{L}^{h} u_{j} \equiv \sum_{\nu=0}^{m} A_{2 \nu}\left(t_{j}\right)\left(D_{+} D_{-}\right)^{\nu} u_{j}+\sum_{\nu=0}^{m-1} A_{2 \nu+1}\left(t_{j}\right)\left(D_{+} D_{-}\right)^{\nu} D_{0} u_{j}=f\left(t_{j}\right)
$$


The boundary conditions are approximated by:

$$
\text { b) } \begin{aligned}
\mathscr{B}_{k}^{h} u^{h} \equiv \sum_{\nu=0}^{m-1}\left\{\left[B_{k, 2 \nu+1}(a)\left(D_{+} D_{-}\right)^{\nu} D_{+} u_{0}+B_{k, 2 \nu+1}(b)\left(D_{+} D_{-}\right)^{\nu} D_{+} u_{J}\right]\right. \\
\left.+\left[B_{k, 2 \nu}(a)\left(D_{+} D_{-}\right)^{\nu} M_{+} u_{0}+B_{k, 2 \nu}(b)\left(D_{+} D_{-}\right)^{\nu} M_{+} u_{J}\right]\right\}=g_{k}, \\
0 \leqq k \leqq n-1 .
\end{aligned}
$$

Recall that on $\pi_{h}^{e}, t_{0}=a-h / 2$ and $t_{J}=b-h / 2$.

For odd $n=2 m+1$ we use $u^{h}$ on the net $\pi_{h}^{o}$ but still approximate (1.1) at each point in $[a, b] \cap \pi_{h}^{e}$ by:

$$
\text { a) } \begin{aligned}
\mathscr{L}^{h} u_{j} & \equiv \sum_{\nu=0}^{m}\left[A_{2 \nu+1}\left(t_{j-1 / 2}\right)\left(D_{+} D_{-}\right)^{\nu} D_{-} u_{j}+A_{2 \nu}\left(t_{j-1 / 2}\right)\left(D_{+} D_{-}\right)^{\nu} M_{-} u_{j}\right] \\
& =f\left(t_{j-1 / 2}\right), \quad 1 \leqq j \leqq J
\end{aligned}
$$

The boundary approximations are now:

$$
\begin{aligned}
& \mathscr{B}_{k}^{k} u^{h} \equiv \sum_{\nu=0}^{m}\left[B_{k, 2 \nu}(a)\left(D_{+} D_{-}\right)^{\nu} u_{0}+B_{k, 2 \nu}(b)\left(D_{+} D_{-}\right)^{\nu} u_{J}\right] \\
&+\sum_{\nu=0}^{m-1}\left[B_{k, 2 \nu+1}(a)\left(D_{+} D_{-}\right)^{\nu} D_{0} u_{0}+B_{k, 2 \nu+1}(b)\left(D_{+} D_{-}\right)^{\nu} D_{0} u_{J}\right]=g_{k}, \\
& 0 \leqq k \leqq n-1 .
\end{aligned}
$$

It follows almost trivially from the definitions (2.8) that the schemes (2.9) and (2.10) are compact and accurate of order 2 . However since they are also centered the truncation errors have asymptotic expansions in powers of $h^{2}$, say in the form:

$$
\begin{aligned}
& \text { a) } \tau_{j}[y]=\sum_{\mu=1}^{L} h^{2 \mu} T_{\mu}\left[y\left(\hat{t}_{j}\right)\right]+\mathcal{O}\left(h^{2 L+2}\right), \quad 1 \leqq j \leqq J \\
& \text { b) } \sigma_{k}[y]=\sum_{\mu=1}^{L} h^{2 \mu} T_{k \mu}[y(a), y(b)]+\mathcal{O}\left(h^{2 L+2}\right), \quad 0 \leqq k \leqq n-1, \\
& \text { where } t_{j}= \begin{cases}t_{j+1 / 2}, & \theta=0, \\
t_{j}, & \theta=e .\end{cases}
\end{aligned}
$$

The detailed forms of these expansions are required for our higher order methods. They can be obtained by formal Taylor expansions or equivalently by using standard finite difference operator identities. We use the latter approach and in terms of the shift operator representation

$$
E(h) \equiv e^{h \mathscr{D}}
$$

it easily follows from (2.8) that:

a) $\quad D_{ \pm}=( \pm h)^{-1}[E( \pm h)-I]=2( \pm h)^{-1} \sinh ( \pm h \mathscr{D} / 2) E( \pm h / 2)$

$$
=\mathscr{D}\left(I+h^{2} \frac{\mathscr{D}^{2}}{4.3 !}+h^{4} \frac{\mathscr{D}^{4}}{16.5 !}+\cdots\right) E( \pm h / 2) \text {, }
$$

b) $M_{ \pm}=\left(I+h^{2} \frac{\mathscr{D}^{2}}{4.2 !}+h^{4} \frac{\mathscr{D}^{4}}{16.4 !}+\cdots\right) E( \pm h / 2)$, 

c) $D_{+} D_{-}=\mathscr{D}^{2}\left(I+h^{2} \frac{\mathscr{D}^{2}}{4.3 !}+h^{4} \frac{\mathscr{D}^{4}}{16.5 !}+\cdots\right)^{2}$,
d) $D_{0}=\mathscr{D}\left(I+h^{2} \frac{\mathscr{D}^{2}}{3 !}+h^{4} \frac{\mathscr{D}^{4}}{5 !}+\cdots\right)$.

Using (2.12) in (2.4) we obtain (2.11) with:
a) $T_{\mu}\left[y\left(\hat{t}_{j}\right)\right]=\sum_{\nu=0}^{n} \alpha_{\mu \nu}^{\theta} A_{\nu}\left(\hat{t}_{j}\right) \mathscr{D}^{\nu+2 \mu} y\left(\hat{t}_{j}\right)$
b) $\quad T_{k \mu}[y(a), y(b)]=\sum_{\nu=0}^{n-1} \beta_{\mu \nu}^{\theta}\left[B_{k, \nu}(a) \mathscr{D}^{\nu+2 \mu} y(a)+B_{k, \nu}(b) \mathscr{D}^{\nu+2 \mu} y(b)\right]$.

The points $\hat{t}_{j} \in \pi_{h}^{e}$ are always on the even net since the difference schemes are centered there for both odd and even order differential equations. The coefficients $\alpha_{\mu \nu}^{\theta}$ and $\beta_{\mu \nu}^{\theta}$ are fixed rational numbers, with $\theta \equiv e(0)$ for $n$ even (odd).

To derive the coefficients we let $D_{2, \theta}^{\nu}$ denote the $\mathscr{O}\left(h^{2}\right)$ approximation to $\mathscr{D}^{\nu}$ on the net $\pi_{h}^{\theta}$ used in (2.9) and (2.10). Then the coefficients $\alpha_{\mu \nu}^{\theta}$ are those in the asymptotic expansion of the form:

$$
D_{2, \theta}^{\nu} y=\mathscr{D}^{\nu} y+\sum_{\mu=1}^{L} \alpha_{\mu \nu}^{\theta} \mathscr{D}^{\nu+2 \mu} y h^{2 \mu}+\mathscr{O}\left(h^{2 L+2}\right) .
$$

The coefficients $\boldsymbol{\beta}_{\mu \nu}^{\theta}$ are simply given by

$$
\beta_{\mu \nu}^{e} \equiv \alpha_{\mu \nu}^{o}, \quad \beta_{\mu \nu}^{o} \equiv \alpha_{\mu \nu}^{e}
$$

[taking some care since there is a difference of a shift operator between (2.9b) and (2.10a)]. These expansions are discussed further in $\$ 2.1$. Tables 1 and 2 give the coefficients $\alpha_{\mu \nu}^{e}, \beta_{\mu \nu}^{e}$ for $\mu=1, \cdots, 6, \nu=0, \cdots, 10$. These are sufficient to perform up to 3 deferred corrections on systems of order $\leqq 4$. The values in these tables were generated using symbolic manipulation via MACSYMA. Details are given in Keller and Pereyra [10].

2.1. Higher order compact schemes. In commonly occurring circumstances we can retain the compactness and $h^{2}$ expansions of the schemes while getting high order accuracy. The best known example of this device is the Collatz Mehrstellenverfahren [1] (a three point scheme for $y^{\prime \prime}=f(t, y)$ which is fourth order accurate). The idea is to use the differential equation, formally, to eliminate or rather approximate the higher derivatives occurring in the truncation errors. To do this while retaining compactness and centering requires certain of the lower order derivatives to be absent from the equation.

In general we claim that: if $A_{\nu}(t) \equiv 0$ for $\nu=n-1, n-2, \cdots, n-2 p+3$ for some integer $p$ in $2 \leqq p<(n+3) / 2$, then a centered compact scheme of accuracy $2 p$ can be obtained. To show this note that when the indicated coefficients vanish any solution of (1.1) satisfies, upon recalling that $A_{n}(t) \equiv I$,

$$
\mathscr{D}^{n} y(t)=f(t)-\sum_{\nu=0}^{n-2 p+2} A_{\nu}(t) \mathscr{D}^{\nu} y(t) .
$$

Using this in (2.13a) we get the representation

$$
T_{\mu}[y(t)]=\alpha_{\mu n}^{\theta} \mathscr{D}^{2 \mu}\left[f(t)-\sum_{\nu=0}^{n-2 p+2} A_{\nu}(t) \mathscr{D}^{\nu} y(t)\right]+\sum_{\nu=0}^{n-2 p+2} \alpha_{\mu \nu}^{\theta} A_{\nu}(t) \mathscr{D}^{\nu+2 \mu} y(t) .
$$


Thus the highest order derivative of $y(t)$ which occurs in $T_{\mu}[y]$ is in the term, for $k=n-2 p+2$ :

$$
\mathscr{L}^{2 \mu, k} y(t) \equiv \mathscr{D}^{2 \mu}\left[A_{k}(t) \mathscr{D}^{k} y(t)\right] .
$$

This and similar terms with $k<n-2 p+2$ must be approximated to accuracy $2(p-\mu)$ using at most $n+1$ points with centered difference approximations and it must be done for $\mu=1,2, \cdots, p-1$. To show that this can be done on each net we use the following basic result.

LEMMA 2.15. Let $\varphi(t)$ be a vector function and $A_{k}(t)$ a matrix function, both sufficiently smooth, and let $h$ be sufficiently small. Let $\mathscr{L}^{2 \mu, k} \varphi(t) \equiv \mathscr{D}^{2 \mu}\left(A_{k}(t) \mathscr{D}^{k} \varphi(t)\right)$. Then, on $\pi_{h}^{e}$ and $\pi_{h}^{o}$ there exist centered difference operators $L_{2 s, e}^{2 \mu, k}, L_{2 s, o}^{2 \mu, k}$, and rational numbers $C_{s, \nu, j}^{2 \mu+k, \theta}$ such that

$$
\text { a) } \quad L_{2 s, \theta}^{2 \mu, k} \varphi\left(t_{j}\right)=\mathscr{L}^{2 \mu, k} \varphi\left(\hat{t}_{j}\right)+\sum_{\nu=s}^{R} h^{2 \nu} \sum_{i=0}^{\nu} C_{s, \nu, i}^{2 \mu, k, \theta} \mathscr{D}^{2 i} \mathscr{L}^{2 \mu, k} \mathscr{D}^{2(\nu-i)} \varphi\left(\hat{t}_{j}\right)+\mathcal{O}\left(h^{2 R+2}\right)
$$

where $\hat{t}_{j} \in[a, b] \cap \pi_{h}^{e}$ and $t_{j} \in \pi_{h}^{\theta}$. Further the number of points $m_{2 s, \theta}^{2 \mu, k}$ used in $L_{2 s, \theta}^{2 \mu, k}$ is given by:

$$
\text { b) } \quad m_{2 s, \theta}^{2 \mu, k}=\left\{\begin{array}{l}
2(\mu+r+s)+\left\{\begin{array}{l}
-1 \\
+1
\end{array}, \text { if } k=\left\{\begin{array}{l}
2 r \\
2 r+1
\end{array}, \quad \theta=e\right.\right. \\
2(\mu+r+s)+1, \quad \text { if } k=\left\{\begin{array}{l}
2 r \\
2 r+1
\end{array}, \quad \theta=o .\right.
\end{array}\right.
$$

Proof. We simply construct these operators recursively. Recalling (2.12c) and taking formal powers we get

$$
D_{2, e}^{2 q} \equiv\left(D_{+} D_{-}\right)^{q}=\mathscr{D}^{2 q}+\sum_{s=1}^{R} h^{2 s} C_{1, s}^{2 q, e} \mathscr{D}^{2(q+s)}+\mathcal{O}\left(h^{2 R+2}\right) .
$$

The rational coefficients $C_{1, s}^{2 q, e}=\alpha_{s, 2 q}^{e}$ and hence can be found in Table 1 . On $\pi_{h}^{e}$ we define, using (2.16):

$$
\begin{aligned}
L_{2, e}^{2 \mu, 2 r} & \equiv D_{2, e}^{2 \mu}\left(A_{2 r} D_{2, e}^{2 r}\right) \\
& =\left[\mathscr{D}^{2 \mu}+\sum_{s=1}^{R} h^{2 s} C_{1, s}^{2 \mu, e} \mathscr{D}^{2(\mu+s)}\right] A_{2 r}\left[\mathscr{D}^{2 r}+\sum_{t=1}^{R} h^{2 t} C_{1, t}^{2 r, e} \mathscr{D}^{2(r+t)}\right] .
\end{aligned}
$$

Upon expanding this expression in powers of $h^{2}$ (being careful with the noncommutative products) we obtain

$$
L_{2, e}^{2 \mu, 2 r}=\mathscr{L}^{2 \mu, 2 r}+\sum_{\nu=1}^{R} h^{2 \nu} \sum_{j=0}^{\nu} C_{1, \nu, j}^{2 \mu, 2 r, e} \mathscr{D}^{2 j} \mathscr{L}^{2 \mu, 2 r} \mathscr{D}^{2(\nu-j)}+O\left(h^{2 R+2}\right) .
$$

Observe that $L_{2, e}^{2 \mu, 2 r}$ uses $(2 \mu+r)+1$ grid points since $D_{2, e}^{2}$ uses three points and each application of $D_{2, e}^{2}$ introduces two new points. The method for constructing $\mathcal{O}\left(h^{4}\right)$ order approximations is to subtract an $\mathcal{O}\left(h^{2}\right)$ approximation of the coefficient of $h^{2}$ in the truncation error expansion (2.18). That is, we define:

$$
L_{4, e}^{2 \mu, 2 r} \equiv L_{2, e}^{2 \mu, 2 r}-h^{2}\left[C_{1,1,0}^{2 \mu, 2 r, e} L_{2, e}^{2 \mu, 2 r} D_{2, e}^{2}+C_{1,1,1}^{2 \mu, 2 r, e} D_{2, e}^{2} L_{2, e}^{2 \mu, 2 r}\right] .
$$

This is clearly an $\mathcal{O}\left(h^{4}\right)$ approximation to $\mathscr{L}^{2 \mu, 2 r}$ which requires $2(\mu+r)+3$ grid points. Moreover using (2.16) and (2.18) the truncation error expansion is obtained in the form:

$$
L_{4, e}^{2 \mu, 2 r}=\mathscr{L}^{2 \mu, 2 r}+\sum_{\nu=2}^{R} h^{2 \nu} \sum_{j=0}^{\nu} C_{2, \nu, j}^{2 \mu, 2 r, e} \mathscr{D}^{2 i} \mathscr{L}^{2 \mu, 2 r} \mathscr{D}^{2(\nu-j)}+O\left(h^{2 R+2}\right)
$$


TABLE 1

The coefficients $\alpha_{\mu \nu}^{e}$. Omitted are the values $\alpha_{\mu_{0}}^{e}=1$. Also recall that $\alpha_{\mu \nu}^{o}=\beta_{\mu \nu}^{e}$ given in Table 2 .

\begin{tabular}{|c|c|c|c|c|c|c|}
\hline$v$ & 1 & 2 & 3 & 4 & 5 & 6 \\
\hline \multirow{2}{*}{1} & 1 & 1 & 1 & 1 & 1 & 1 \\
\hline & 6 & 120 & 5040 & 362880 & 39916800 & 6227020800 \\
\hline \multirow{2}{*}{2} & 1 & 1 & 1 & 1 & 1 & 1 \\
\hline & 12 & 360 & 20160 & 1814400 & 239500800 & 43589145600 \\
\hline \multirow{2}{*}{3} & 1 & 1 & 17 & 31 & 1 & 5461 \\
\hline & 4 & 40 & 12096 & 604800 & 760320 & 217945728000 \\
\hline \multirow{2}{*}{4} & 1 & 1 & 17 & 31 & 1 & 5461 \\
\hline & 6 & 80 & 30240 & 1814400 & 2661120 & 871782912000 \\
\hline \multirow{2}{*}{5} & $\underline{1}$ & 7 & 4 & 13 & 31 & 63047 \\
\hline & 3 & 144 & 945 & 51840 & 2851200 & 174356582400 \\
\hline \multirow{2}{*}{6} & $\underline{1}$ & 7 & 2 & 13 & 31 & 63047 \\
\hline & 4 & 240 & 945 & 120960 & 7603200 & 523069747200 \\
\hline \multirow{2}{*}{7} & 5 & 19 & 1 & 457 & 491 & 164573 \\
\hline & 12 & $\overline{240}$ & $\overline{108}$ & 604800 & $\overline{10644480}$ & $\overline{74724249600}$ \\
\hline \multirow{2}{*}{8} & $\underline{1}$ & 19 & 1 & 457 & 491 & 164573 \\
\hline & 3 & 360 & 189 & 1209600 & 23950080 & 186810624000 \\
\hline \multirow{2}{*}{9} & 1 & 7 & 43 & 713 & 317 & 7141 \\
\hline & 2 & 60 & 2520 & 403200 & 2280960 & 825552000 \\
\hline \multirow{2}{*}{10} & 5 & 1 & 43 & 713 & 317 & 7141 \\
\hline & 12 & 12 & 4032 & 725760 & 4561920 & 1816214400 \\
\hline
\end{tabular}

The general result (2.15a) follows by induction on $s$ and the recursive definition of the operators $L_{2 s, e}^{2 m, k}$. For $k=2 r$ we let

$$
L_{2(s+1), e}^{2 \mu, 2 r} \equiv L_{2 s, e}^{2 \mu, 2 r}-h^{2 s} \sum_{j=0}^{s} C_{s, s, j}^{2 \mu, 2 r, e} D_{2, e}^{2 j} L_{2, e}^{2 \mu, 2 r} D_{2, e}^{2(s-j)}
$$

Then $L_{2(s+1), e}^{2 \mu, 2 r}$ uses $[2(\mu+r+s)+1]$ grid points agreeing with the first entry in $(2.15 \mathrm{~b})$ and a simple but somewhat tedious verification shows that $(2.15 \mathrm{a})$ holds for $k=2 r$ and $\theta=e$. For odd $k=2 r+1$ on $\pi_{h}^{e}$ we simply note, using $(2.12 \mathrm{c}, \mathrm{d})$ that

$$
D_{2, e}^{2 r+1} \equiv\left(D_{+} D_{-}\right)^{r} D_{0}=\mathscr{D}^{2 r+1}+\sum_{s=1}^{R} h^{2 s} C_{1, s}^{2 r+1, e} \mathscr{D}^{2(r+s)+1}+O\left(h^{2 R+2}\right),
$$

then defining

$$
L_{2, e}^{2 \mu, 2 r+1} \equiv D_{2, e}^{2 \mu}\left(A_{2 r+1} D_{2, e}^{2 r+1}\right)
$$

the whole procedure goes through as above. Since $D_{2, e}^{2 r+1}$ uses $(2 r+3)$ grid points, $L_{2, e}^{2 \mu, 2 r+1}$ will require $(2 \mu+r)+3$ grid points, and in general $L_{2 s, e}^{2 \mu, 2 r+1}$ will require $2(\mu+r+s)+1$ grid points.

The corresponding results on the odd grid, $\pi_{h}^{o}$, are obtained very similarly using $(2.12 \mathrm{a}, \mathrm{b}, \mathrm{c})$ in $D_{2,0}^{2 q+1} \equiv\left(D_{+} D_{-}\right)^{q} D_{-}$and $D_{2,0}^{2 q} \equiv\left(D_{+} D_{-}\right)^{q} M_{-}$. 
Now we rewrite (2.14) as

$$
T_{\mu}[y]=\alpha_{\mu n}^{\theta} \mathscr{D}^{2 \mu} f(t)-\alpha_{\mu n}^{\theta} \sum_{\nu=0}^{n-2 p+2} \mathscr{D}^{2 \mu}\left(A_{\nu}(t) \mathscr{D}^{\nu} y\right)+\sum_{\nu=0}^{n-2 p+2} \alpha_{\mu, \nu}^{\theta} A_{\nu}(t) \mathscr{D}^{\nu+2 \mu} y .
$$

We will show that by using Lemma 2.15 we can define a compact approximation $T_{p, \mu}^{h}\left[y\left(t_{j}\right)\right]$ to $T_{\mu}\left[y\left(t_{j}\right)\right]$ of accuracy $h^{2(p-\mu)}$ for $1 \leqq \mu \leqq p-1$. Then forming

$$
\text { a) } \mathscr{L}_{p}^{h} u_{j} \equiv \mathscr{L}^{h} u_{j}-\sum_{\mu=1}^{p-1} h^{2 \mu} T_{p, \mu}^{h}[u, j], \quad 1 \leqq j \leqq J,
$$

we will have a compact approximation to $\mathscr{L}$ of order $2 p$. This is all justified by observing, from Lemma 2.15 , that the highest order operator appearing in $(2.20), \mathscr{L}^{2 \mu, n-2(p-1)} y$, can be approximated to order $h^{2(p-\mu)}$ by $L_{2(p-\mu), \theta}^{2 \mu, 2(p-1)} y$ which uses, according to (2.15b), $(n+1)$ net points. The terms involving $\mathscr{D}^{\nu+2 \mu} y$ offer no new difficulty, being special cases of $\mathscr{L}^{2 \mu, \nu} y(t)$.

To actually construct the indicated operators involves considerable manipulation with series, or more accurately with high degree polynomials. We have also done this using symbolic manipulation programs and the details with complete tables of coefficients are contained in Keller and Pereyra [10].

TABLE 2

\begin{tabular}{|c|c|c|c|c|c|c|}
\hline$v$ & 1 & 2 & 3 & 4 & 5 & 6 \\
\hline \multirow{2}{*}{0} & $\underline{1}$ & 1 & 1 & 1 & 1 & 1 \\
\hline & 8 & 384 & 46080 & 10321920 & 3715891200 & 1961990553600 \\
\hline \multirow{2}{*}{1} & 1 & 1 & 1 & 1 & 1 & 1 \\
\hline & 24 & 1920 & 322560 & 92897280 & 40874803200 & 25505877196800 \\
\hline \multirow{2}{*}{2} & 5 & 91 & 41 & 7381 & 949 & 597871 \\
\hline & 24 & 5760 & 64512 & 464486400 & 3503554560 & 178541140377600 \\
\hline \multirow{2}{*}{3} & 1 & 13 & 41 & 671 & 73 & 597871 \\
\hline & 8 & 1920 & 193536 & 154828800 & 1167851520 & $\overline{892705701888000}$ \\
\hline \multirow{2}{*}{4} & 7 & 23 & 2497 & 8203 & 11617 & 99256999 \\
\hline & 24 & 640 & $\overline{967680}$ & 66355200 & 2724986880 & 892705701888000 \\
\hline \multirow{2}{*}{5} & 5 & 23 & 227 & 631 & 11617 & 5838647 \\
\hline & 24 & 1152 & 193536 & 13271040 & $\overline{8174960640}$ & 178541140377600 \\
\hline \multirow{2}{*}{6} & $\underline{3}$ & 121 & 6227 & 4681 & 322643 & 513602813 \\
\hline & 8 & 1920 & 967680 & 10321920 & 13624934400 & $\overline{535623421132800}$ \\
\hline \multirow{2}{*}{7} & 7 & 77 & 479 & 4681 & 18979 & 27031727 \\
\hline & 24 & 1920 & 138240 & 22118400 & 1946419200 & 76517631590400 \\
\hline \multirow{2}{*}{8} & 11 & 559 & 2473 & 183311 & 2027813 & 158165129 \\
\hline & 24 & 5760 & 193536 & 154828800 & 24524881920 & 34780741632000 \\
\hline \multirow{2}{*}{9} & 3 & 43 & 2473 & 10783 & 106727 & 158165129 \\
\hline & 8 & 640 & 322560 & 17203200 & 2724986880 & 81155063808000 \\
\hline \multirow{2}{*}{10} & 13 & 53 & 7157 & 236113 & 1295803 & 183490849 \\
\hline & $\overline{24}$ & $\overline{384}$ & 322560 & $\overline{92897280}$ & $\overline{5839257600}$ & $\overline{11902742691840}$ \\
\hline
\end{tabular}

The coefficients $\beta_{\mu \nu}^{e}$. Also recall that $\beta_{\mu \nu}^{o}=\alpha_{\mu \nu}^{e}$ given in Table 1 . 
A similar treatment of the boundary operators is possible when appropriate lower order derivatives are absent from the differential equation as well as the boundary conditions. It should be recalled here however that the boundary difference approximations need not be compact and thus we have more flexibility in approximating them to higher order. However in close analogy with the above we can define

$$
\text { b) } \mathscr{B}_{p, k}^{h} u^{h} \equiv \mathscr{B}_{k}^{h} u^{h}-\sum_{\mu=1}^{p-1} h^{2 \mu} T_{p, k, \mu}^{h}\left[u_{0}, u_{J}\right], \quad 0 \leqq k \leqq n-1 .
$$

The truncation expansions for these higher order accurate schemes now have the forms, with appropriately defined $T_{p, \nu}[\cdot] ; T_{p, k, \nu}[\cdot, \cdot]$ :
a) $\tau_{p, j}[y]=h^{2 p} \sum_{\nu=0}^{L-p} h^{2 \nu} T_{p, \nu}\left[y\left(\hat{t}_{j}\right)\right]+\mathcal{O}\left(h^{2(L+1)}\right), \quad 1 \leqq j \leqq J$
b) $\sigma_{p, k}[y]=h^{2 p} \sum_{\nu=0}^{L-p} h^{2 \nu} T_{p, k, \nu}[y(a), y(b)]+\mathcal{O}\left(h^{2 L+2}\right), \quad 0 \leqq k \leqq n-1$.

3. Semi-linear systems. The methods and theory of $\S 2$ are easily extended to semi-linear boundary problems of the form:
a) $\mathcal{N} y(t) \equiv \mathscr{L} y(t)-f(t, y(t))=0, \quad \mathrm{a} \leqq \mathrm{t} \leqq \mathrm{b}$;
b) $\mathcal{N}_{k} y \equiv \mathscr{B}_{k} y-g_{k}(y(a), y(b))=0, \quad k=0,1, \cdots, n-1$.

We assume that (3.1) has an isolated solution, $y(t)$, on $[a-\delta, b+\delta]$. That is the linearized problem about $y(t)$ :

a) $\mathscr{L}[y] v(t) \equiv \mathscr{L} v(t)-F(t) v(t)=0, \quad a \leqq t \leqq b$;

b) $\mathscr{B}_{k}[y] v \equiv \mathscr{B}_{k} y-G_{k}(a) y(a)-G_{k}(b) y(b)=0, \quad k=0,1, \cdots, n-1$;

has only the trivial solution, $v(t) \equiv 0$. Here we have introduced the $d \times d$ Jacobian matrices:

c) $\quad F(t) \equiv \frac{\partial f(t, y(t))}{\partial y} ; \quad G_{k}(x) \equiv \frac{\partial g_{k}(y(a), y(b))}{\partial y(x)}, \quad x=a, b, \quad 0 \leqq k \leqq n-1$.

These matrices are also required to be Lipschitz continuous in $y$ near $y(t)$; that is for some $\rho>0$ and $K_{L}>0$ :
a) $\left\|\frac{\partial f(t, y(t))}{\partial y}-\frac{\partial f(t, z(t))}{\partial z}\right\| \leqq K_{L}\|y(t)-z(t)\|$,
b) $\left\|\frac{\partial g_{k}(y(a), y(b))}{\partial y(x)}-\frac{\partial g_{k}(z(a), z(b))}{\partial z(x)}\right\| \leqq K_{L}(\|y(a)-z(a)\|+\|y(b)-z(b)\|)$,

for all $\{t, z(t)\}$ satisfying:
c) $a-\delta \leqq t \leqq b+\delta$,
$\|y(t)-z(t)\| \leqq \rho$.

On the nets $\pi_{h}^{\theta}$ we consider the difference schemes:
a) $\quad \mathcal{N}^{h} u_{j} \equiv \mathscr{L}^{h} u_{j}-\left\{\begin{array}{c}f\left(t_{j}, u_{j}\right) \\ f\left(t_{j-1 / 2}, M_{-} u_{j}\right)\end{array}\right\}=0, \quad n\left\{\begin{array}{c}\mathrm{EVEN} \\ \mathrm{ODD} ; \quad 1 \leqq j \leqq J ;\end{array}\right.$
b) $\mathcal{N}_{k}^{h} u^{h} \equiv \mathscr{B}_{k}^{h} u^{h}-\left\{\begin{array}{c}g_{k}\left(M_{+} u_{0}, M_{+} u_{J}\right) \\ g_{k}\left(u_{0}, u_{J}\right)\end{array}\right\}=0, \quad n\left\{\begin{array}{l}\mathrm{EVEN} \\ \mathrm{ODD}\end{array} ; 0 \leqq k \leqq n-1\right.$ 
To study these difference schemes we need the basic stability result in

LEMMA 3.5. Let (3.1) have the isolated solution $y(t)$ for which (3.3) holds. Then for $h_{0}$ and $\rho$ sufficiently small and on the family of nets $\pi_{h}^{\theta}$ with $h \leqq h_{0}$, for all net-functions $v^{h} \equiv\left\{v_{j}\right\}, w^{h} \equiv\left\{w_{j}\right\}$ with $\left\|v^{h}-y^{h}\right\|=\max \left\|v_{j}-y\left(t_{j}\right)\right\| \leqq \rho$ and $\left\|w^{h}-y^{h}\right\| \leqq \rho:$

$$
\left\|v^{h}-w^{h}\right\| \leqq K_{1}\left\{\max _{1 \leqq j \leqq J}\left\|\mathcal{N}^{h} v_{j}-\mathcal{N}^{h} w_{j}\right\|+\max _{0 \leqq k<n}\left\|\mathcal{N}_{k}^{h} v^{h}-\mathcal{N}_{k}^{h} w^{h}\right\|\right\}
$$

Proof. The linear difference schemes $\left\{\hat{\mathscr{D}}^{h}, \hat{\mathscr{B}}_{k}^{h}\right\}$ are stable where $\mathscr{L}^{h}$ is obtained from $\mathscr{L}^{h}$ in (2.9a) or (2.10a) by replacing $A_{0}(t)$ by $A_{0}(t)-F(t)$ and the $\mathscr{\mathscr { B }}_{k}^{h}$ are obtained from $\mathscr{B}_{k}^{h}$ in (2.9b) or (2.10b) by replacing $B_{k, 0}(x)$ by $B_{k, 0}(x)-G_{k}(x), x=a, b, 0 \leqq k \leqq$ $n-1$. This follows from Theorem 2.7 since $\left\{\mathscr{\mathscr { L }}^{h}, \hat{\mathscr{B}}_{k}^{h}\right\}$ are compact schemes consistent with (3.2) which has a unique solution.

Thus the net function $v^{h}-w^{h}$ satisfies for some $K$ and $h_{0}$ :

$$
\left\|v^{h}-w^{h}\right\| \leqq K\left\{\max _{1 \leqq j \leqq J}\left\|\hat{\mathscr{L}}^{h}\left(v_{j}-w_{j}\right)\right\|+\max _{0 \leqq k<n}\left\|\hat{\mathscr{B}}_{k}^{h}\left(v^{h}-w^{h}\right)\right\|\right\} .
$$

However $\mathscr{L}^{h} v_{j} \equiv \mathscr{L}^{h} v_{j}-F\left(t_{j}\right) v_{j}$ for $n$ even and $\mathscr{L}^{h} v_{j} \equiv \mathscr{L}^{h} v_{j}-F\left(t_{j-1 / 2}\right) M_{-} v_{j}$ for $n$ odd, with similar expressions for the boundary terms. Thus we may use the definitions (3.4) to get for $n$ even:

$$
\begin{aligned}
\left\|v^{h}-w^{h}\right\| \leqq K\left\{\max _{j}\left\|\left[\mathcal{N}^{h} v_{j}-F\left(t_{j}\right) v_{j}+f\left(t_{j}, v_{j}\right)\right]-\left[\mathcal{N}^{h} w_{j}-F\left(t_{j}\right) w_{j}+f\left(t_{j}, w_{j}\right)\right]\right\|\right. \\
+\sum_{k=0}^{n-1} \|\left[\mathcal{N}_{k}^{h} v^{h}-G_{k}(a) M_{+} v_{0}-G_{k}(b) M_{+} v_{J}+g_{k}\left(M_{+} u_{0}, M_{+} u_{J}\right)\right] \\
\left.\quad-\left[\mathcal{N}_{k}^{h} w^{h}-G_{k}(a) M_{+} w_{0}-G_{k}(b) M_{+} w_{J}+g_{k}\left(M_{+} w_{0}, M_{+} w_{J}\right)\right] \|\right\} .
\end{aligned}
$$

From (3.2c) and (3.3a) it follows, since $v^{h}$ and $w^{h}$ are within $\rho$ of $y^{h}$, that

$$
\left\|f\left(t_{j}, v_{j}\right)-f\left(t_{j}, w_{j}\right)-F\left(t_{j}\right)\left(v_{j}-w_{j}\right)\right\| \leqq \rho K_{L}\left\|v_{j}-w_{j}\right\| .
$$

Similar estimates hold for the boundary terms and thus we get:

$$
\left(1-n K K_{L} \rho\right)\left\|v^{h}-w^{h}\right\| \leqq K\left\{\max _{j}\left\|\mathcal{N}^{h} v_{j}-\mathcal{N}_{w_{j}}^{h}\right\|+\max _{k}\left\|\mathcal{N}_{k}^{h} v^{h}-\mathcal{N}_{k}^{h} w^{h}\right\|\right\} .
$$

If $\rho$ is so small that $n K K_{L} \rho<1$ then (3.5) follows with $K_{1}=K /\left(1-n K K_{L} \rho\right)$.

The case of $n$ odd is treated in a similar manner.

With stability in the form (3.5) we can easily prove that the nonlinear difference equations (3.4) have, for $h_{0}$ and $\rho$ sufficiently small, a unique solution $u^{h}$ within $\rho$ of $y^{h}$. Indeed since (3.4) is second order accurate we have that $\left\|u^{h}-y^{h}\right\|=\mathscr{O}\left(h^{2}\right)$. Further the numerical solution can be computed using Newton's method which converges quadratically (for an appropriate initial guess). These results are easily proven using the techniques of $\S 4$ in [11] so we do not repeat them here.

The truncation errors of the schemes in (3.4) still retain the $h^{2}$-expansions of the linear case. Indeed the higher order accurate theory of $\S 2.1$ goes over with no essential change to define the $\mathcal{N}_{p}^{h}$ and $\mathcal{N}_{p, k}^{h}, 0 \leqq k \leqq n-1$. The expansions of the nonlinearities yield many extra terms but no theoretical difficulties are encountered. The truncation errors retain the forms indicated in (2.13).

4. Deferred corrections. As we have shown in $\S 2$ and $\S 3$ centered compact difference schemes of the form (2.2) can be devised which have, for some $p \geqq 1$, local truncation error expansions of the form

$$
\text { a) } \tau_{p, j}[y]=h^{2 p} \sum_{\nu=0}^{L-p} h^{2 \nu} T_{p, \nu}\left[y\left(\hat{t}_{j}\right)\right]+\mathcal{O}\left(h^{2(L+1)}\right), \quad 1 \leqq j \leqq J
$$




$$
\text { b) } \sigma_{p, k}[y]=h^{2 p} \sum_{\nu=0}^{L-p} h^{2 \nu} T_{p, k, \nu}[y(a), y(b)]+\mathcal{O}\left(h^{2(L+1)}\right), \quad 0 \leqq k \leqq n-1 .
$$

Since the schemes are stable it then easily follows that the global errors have corresponding asymptotic expansions of the form:

$$
u_{j}-y\left(t_{j}\right)=h^{2 p} \sum_{\nu=0}^{L-p} h^{2 \nu} e_{\nu}\left(t_{j}\right)+\mathcal{O}\left(h^{2(L+1)}\right) .
$$

Here the $e_{\nu}(t)$ are solutions of linear two point boundary value problems similar to (1.1)-(1.2) or (3.2) but with inhomogeneous data determined by the truncation operators on $y(t)$ and the $e_{\mu}(t)$ for $\mu<\nu$. These derivations are by now standard, see for example [5], [14], [19].

Richardson extrapolation is justified by (4.2) and yields two orders of accuracy improvement per application. This procedure essentially eliminates successive terms, $e_{\nu}(t)$, in (4.2) by forming appropriate linear combinations of the solutions of the same centered compact scheme applied on successively refined nets.

The deferred correction method uses a sequence of altered difference schemes in which sets of successive terms of the local truncation errors (4.1) are eliminated by using solutions of the previous scheme. More precisely the method defines a sequence of net functions $\left\{u_{j}(q)\right\}$ which have global errors of the form:

$$
\begin{aligned}
u_{j}(q)-y\left(t_{j}\right)=h^{2 p q} & \sum_{\nu=0}^{L-q p} h^{2 \nu} e_{\nu}\left(q ; t_{j}\right)+\mathcal{O}\left(h^{2(L+1)}\right) \\
& -\underline{m} \leqq j \leqq J+m ; \quad q=1,2, \cdots, L / p .
\end{aligned}
$$

To define these net functions we require difference approximations $S_{p q}[\cdot], S_{p k q}[\cdot, \cdot]$ to the truncation operators in (4.1) which have the properties that when (4.3) holds then:

$$
\begin{aligned}
& \text { a) } S_{p, q}\left[u_{j}(q-1)\right]=h^{2 p} \sum_{\nu=0}^{p q} h^{2 \nu} T_{p, \nu}\left[y\left(\hat{t}_{j}\right)\right]+\mathcal{O}\left(h^{2 p(q+1)}\right), \quad 1 \leqq j \leqq J \\
& \text { b) } S_{p, k, q}\left[u^{h}(q-1)\right]=h^{2 p} \sum_{\nu=0}^{p q} h^{2 \nu} T_{p, k, \nu}[y(a), y(b)]+\mathcal{O}\left(h^{2 p(q+1)}\right)
\end{aligned}
$$

$$
0 \leqq k \leqq n-1 .
$$

Recursively starting with $u_{j}(1) \equiv u_{j}$, the original $\mathcal{O}\left(h^{2 p}\right)$ accurate solution of (2.2) say, we then define $\left\{u_{j}(q)\right\}$ as the solution of:
a) $\mathscr{L}_{p}^{h} u_{j}(q)-f^{h}\left(t_{j}\right)=S_{p, q}\left[u_{j}(q-1)\right], \quad 1 \leqq j \leqq J$.
b) $\mathscr{B}_{p, k}^{h} u^{h}(q)-g_{k}^{h}=S_{p, k, q}\left[u^{h}(q-1)\right], \quad 0 \leqq k \leqq n-1$.

From stability and (4.4) it easily follows by an induction that (4.3) holds. Note that for the semi-linear case we need only replace the left hand sides in (4.5) by the difference operators in (3.4). Thus to justify and use deferred corrections we must determine difference operators satisfying (4.4).

Before turning to this basic problem we note the main features which distinguish deferred corrections (DC) from Richardson extrapolation (RE):

i) DC always employs a fixed net so that the dimensionality of the linear systems to be solved at each correction (or Newton iterate in the nonlinear cases) is also fixed.

ii) For the linear case only the inhomogeneous terms change for each correction and thus only one $L U$-decomposition of a large block-structured matrix need be done. 
For the nonlinear cases using Newton's method to solve the difference equations the Jacobian matrices remain of fixed size and indeed change very little from one correction to the next. Use can be made of this to enhance the efficiency of the solution procedure by switching over to the chord method (or "special Newton method") with the last $L U$-decomposed Jacobian as the fixed "slope" matrix.

iii) Since $2 p$ orders of accuracy are gained in DC on each correction, very few need be done for $p \geqq 2$.

iv) Special pains must be taken in DC to compute the approximating truncation operators (4.4) so that they retain the $h^{2}$-expansion property and requisite order of accuracy. This problem is somewhat simplified by one of the devices explained in $\S 5$.

v) RE uses only one basic scheme but with a variable number of net points. Thus while the code is easily written, successive extrapolations take many more operations. It gains only two orders of accuracy per extrapolation.

5. Approximate truncation operators; boundary difficulties. An obvious way in which the approximate truncation operator $S_{p, q}[\cdot], S_{p, k, q}[\cdot, \cdot]$ of $(4.4)$ might be defined is to use centered difference operators to approximate all the desired derivatives. The trouble of course is that this can only be done sufficiently far from the endpoints of the interval. As the boundaries are approached one must employ noncentered difference approximations and thus the $h^{2}$-expansion property is lost. Also the usual derivations of asymptotic error expansions of the form (4.3) fail in this case since the inhomogeneous terms required to define the $e_{\nu}(q, t)$ are discontinuous. These difficulties are discussed in [17] where special nonsymmetric endpoint formulas are presented along with appropriate weight generators. The performance of this procedure using noncentered differences has shown deviations from the theoretically expected behavior.

Two alternate treatments of the boundary difficulties have been independently proposed by O. Widlund [18] and by $\mathrm{H}$. B. Keller [9]. Both retain the use of centered difference operators in constructing the $S_{p, q}[\cdot]$ by extending the difference solutions outside the basic interval $[a, b]$. Widlund does this by extrapolating the computed solution from the interior by means of appropriate high order accurate extrapolation formulas. The procedure is subject to magnification of errors if very high order extrapolation is required. Indeed it is closely related to Pereyra's treatment [17] since it must be equivalent to the use of some unsymmetric difference operators applied to the internal data. Thus it cannot retain $h^{2}$-expansions to all orders. However it is conceptually simple and relatively easy to implement.

Keller's treatment [9] is to pose and solve the boundary value problem over a slightly enlarged interval interior to $[a-\delta, b+\delta]$ but exterior to $[a, b]$. Thus we consider in place of (3.1) (or (1.1)-(1.2)):

$$
\begin{aligned}
& \text { a) } \mathcal{N} y_{x}(t) \equiv \mathscr{L}_{x}(t)-f\left(t, y_{x}(t)\right)=0, \quad a-\delta \leqq t \leqq b+\delta \\
& \text { b) } \mathcal{N}_{k} y_{x} \equiv \mathscr{B}_{k} y_{x}-g_{k}\left(y_{x}(a), y_{x}(b)\right)=0, \quad 0 \leqq k \leqq n-1 .
\end{aligned}
$$

Here we assume that the original problem has an isolated solution, $y(t)$ on $[a, b]$ and extend, with appropriate smoothness, the $A_{\nu}(t)$ and $f(t, y)$ to the enlarged interval. Then the extended problem has an isolated solution, $y_{x}(t)$, on $[a-\delta, b+\delta]$. This solution coincides with that of the original problem on $[a, b]$ i.e., $y(t) \equiv y_{x}(t)$ for $a \leqq t \leqq b$. Exterior to $[a, b]$ the solution, $y_{x}(t)$, of $(5.1)$ can be defined by the initial value problems:

$$
\begin{cases}\mathscr{L} y_{L}(t)=f\left(t, y_{L}(t)\right), & a-\delta \leqq t \leqq a \\ \mathscr{D}^{k} y_{L}(a)=\mathscr{D}^{k} y(a), & 0 \leqq k \leqq n-1\end{cases}
$$


and

$$
\begin{cases}\mathscr{L} y_{R}(t)=f\left(t, y_{R}(t)\right), & b \leqq t \leqq b+\delta \\ \mathscr{D}^{k} y_{R}(b)=\mathscr{D}^{k} y(b), & 0 \leqq k \leqq n-1\end{cases}
$$

These problems must be solved numerically using the same difference schemes as are used over $[a, b]$. Now however they are used as "shooting" or initial-value schemes but only over intervals of length $r_{0} h<\delta$. The asymptotic error expansions retain the same form as those in the interior of $[a, b]$ and hence the justification of deferred corrections is assured to all orders. We indicate more details below.

5.1. The extension procedure for deferred corrections. Suppose $\left\{u_{j}(1)\right\}, \cdots,\left\{u_{j}(q-1)\right\}$ have been determined so that (4.3) holds on some extended net. Then to determine $\left\{u_{j}(q)\right\}$ we must show how to construct the $S_{p, q}[\cdot]$ and $S_{p, k, q}[\cdot]$ on an appropriate net somewhat reduced from the extended net. We shall use some of the results and techniques introduced in $\S 2.1$ to do this. First note from (4.4) that $S_{p, q}[\cdot]$ only requires that $T_{p, \nu}\left[y\left(\hat{t}_{j}\right)\right]$ be approximated to accuracy $h^{2(p q-\nu)}$ for $\nu=$ $0,1, \cdots, q p-1$ using $\left\{u_{j}(q-1)\right\}$. The highest derivative occurring in $T_{p, \nu}[y(t)]$ is $\mathscr{D}^{n+2(\nu-p+1)} y(t)$. This and all other terms in $T_{p, \nu}[\cdot]$ must be approximated to accuracy $h^{2(p q-\nu)}$. We can make these approximations by using the difference operators $D_{2 s, \theta}^{k}$ of Lemma 2.15. In particular $D_{2(p q-\nu), \theta}^{n+2(\nu-p+1)}$ uses $[n+2 p(q-1)+1]$ points since $\theta$ is determined by the parity of $n$ [i.e., $\theta=e$ iff $n$ is even, see (2.15b)]. Note that this number of points suffices for all required $\nu$ in $0 \leqq \nu \leqq q p-1$ and is indepent of $\nu$. Further as shown in Lemma 2.15 all lower order derivatives appearing in any of the $T_{p, \nu}[\cdot]$ can be approximated to the required accuracy using the same or fewer number of points.

Now recall that our basic compact schemes employ $n+1$ netpoints in each stencil about the points $\hat{t}_{j} \in[a, b] \cap \pi_{h}^{e}$. Since we employ only centered differences throughout, our correction terms use $p(q-1)$ additional points on each side of the basic stencils [to give the total of $n+1+2 p(q-1)$ points]. Thus $u_{j}(q-1)$ must be computed on $p(q-1)$ points beyond either end of the mesh interval on which $u_{j}(q)$ is to be computed. By induction on $q$ we now find that: for each $k$ in $1 \leqq k \leqq q$ the corrected solutions $\left\{u_{j}(k)\right\}$ with accuracy $h^{2 p k}$ must be computed on $\left\{t_{j}\right\}$ with:

$$
\begin{aligned}
& \text { a) }-\underline{m}-r_{k, q} \leqq j \leqq J+m+r_{k, q} \text {, } \\
& \text { b) } r_{k, q}=[q(q-1)-k(k-1)] p / 2, \quad 1 \leqq k \leqq q .
\end{aligned}
$$

The initial value problems (5.2) need only be computed on the $r_{k, q}$ points beyond $t_{-m}$ and $t_{J+m}$. The stability of this shooting procedure is assured since the number of points is fixed independently of the net spacing, $h$.

6. Implementation of deferred corrections. We have seen in the sections above how to generate high order approximations to a fairly general class of boundary value problems. The simplest approach to actually implementing these ideas in a computer code requires the consideration of two separate cases according to the parity of $n$, the system's order.

We explain in some detail how an even order deferred correction solver has been devised. We consider only the general case $p=1$ [i.e. the basic scheme is $\mathscr{O}\left(h^{2}\right)$ ].

Recalling formulas (2.2) we require the coefficients $C_{s}\left(t_{j}, h\right), C_{k s}(a, h), C_{k s}(b, h)$. 
Clearly the $\mathscr{O}\left(h^{2}\right)$ approximations $D_{2, e}^{\nu}$ to $\mathscr{D}^{\nu}$ used in (2.9) are of the form

$$
\begin{aligned}
& D_{2, e}^{2 \nu} u_{i}=h^{-2 \nu} \sum_{s=-\nu}^{\nu} w_{s}^{2 \nu, e} u_{i+s}, \\
& D_{2, e}^{2 \nu+1} u_{i}=h^{-(2 \nu+1)} \sum_{s=-(\nu+1)}^{\nu+1} w_{s}^{2 \nu+1, e} u_{i+s} .
\end{aligned}
$$

Symbolic procedures for generating the weights $w_{s}^{\nu, e}$ and complete tables of them for a wide range of derivatives are given in [10]. Of course, fast Vandermonde solvers [19] can also be used to generate these weights if storage space is at a premium.

Since

$$
\mathscr{L}^{h} u_{j}=\sum_{\nu=0}^{n} A_{\nu}\left(t_{j}\right) D_{2, e}^{\nu} u_{j},
$$

we have, by using the above expressions

$\mathscr{L}^{h} u_{j}=\sum_{\nu=0}^{m} A_{2 \nu}\left(t_{j}\right)\left(\sum_{s=-\nu}^{\nu} w_{s}^{2 \nu, e} u_{j+s}\right) h^{-2 \nu}+\sum_{\nu=0}^{m-1} A_{2 \nu+1}\left(t_{j}\right)\left(\sum_{s=-(\nu+1)}^{\nu+1} w_{s}^{2 \nu+1, e} u_{j+s}\right) h^{-(2 \nu+1)}$.

Thus the coefficients for (2.2a) are with $C_{s j} \equiv C_{s}\left(t_{j}, h\right)$ :

$$
C_{s j}=w_{s}^{n, e} h^{-n} I+\sum_{\nu=|s|}^{m-1} w_{s}^{2 \nu, e} h^{-2 \nu} A_{2 \nu}\left(t_{j}\right)
$$

$$
+\sum_{\nu=\max (0,|s|-1)}^{m-1} w_{s}^{2 \nu+1, e} h^{-(2 \nu+1)} A_{2 \nu+1}\left(t_{j}\right), \quad s=-m, \cdots, m .
$$

In this formula, $I$ is the $d \times d$ identity matrix. A similar computation in (2.9b) yields for the coefficients in $(2.2 \mathrm{~b})$ :

$$
\begin{aligned}
& C_{k s}(x, h)=\sum_{\nu=|s-1 / 2|-1 / 2}^{m-1} h^{-(2 \nu+1)}\left(B_{k, 2 \nu+1}(x) w_{s}^{2 \nu+1, o}+h B_{k, 2 \nu}(x) w_{s}^{2 \nu, o}\right), \\
& s=-\underline{m}, \cdots, m ; \quad x=a, b ; \quad k=1, \cdots, n \times d .
\end{aligned}
$$

Here $w_{s}^{\nu, o}$ are the weights corresponding to the $O\left(h^{2}\right)$ approximations to $\mathscr{D}^{v}$ on an odd mesh.

With the known coefficient matrices $C_{s j}, C_{k s}(a, h), C_{k s}(b, h)$ we obtain a matrix representation of the linear system (2.2) in the form

$$
\mathbb{A} u^{h}=f^{h} \text {. }
$$

For simplicity we have only considered in our implementation the separated boundary condition case, where there are $p$ "initial" conditions at $t=a$ and $(n d-p)$ "end" conditions at $t=b$. Writing the equations for the initial conditions at the beginning and those for the end conditions as the last equations, the matrix $\mathbb{A}$ of $(6.2)$ has the block form

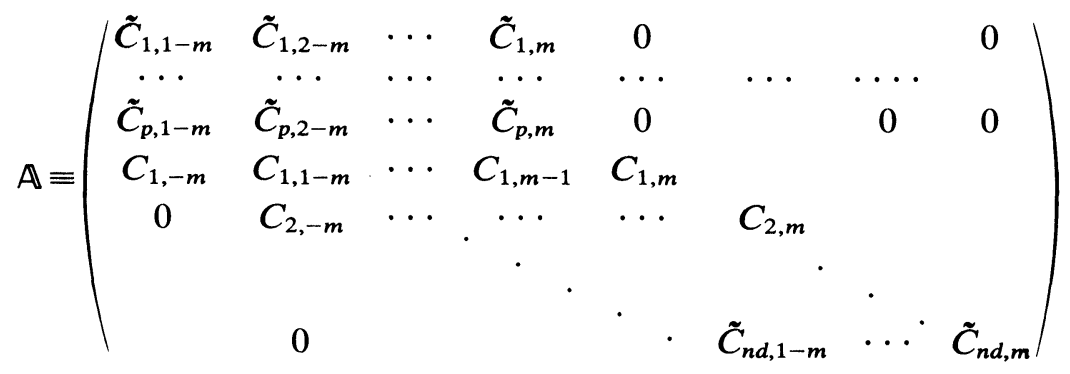


When written in this form $A$ is a band matrix with half band width $\beta$ given by

$$
\beta=\max [n d, p+d,(n+1) d-p]-1 .
$$

To obtain the basic $\mathcal{O}\left(h^{2}\right)$ accuate solution $\left\{u_{j}(1)\right\}$ on the net $\left\{t_{j}\right\}, 1-m \leqq j \leqq J+m$, we need only sove the system (6.2) with coefficient matrix (6.3).

Extension procedure. The extension procedure, shooting from both ends in order to provide the additional external points to use in the centered deferred correction formulas, proceeds as follows. After the $q$ th correction $\left\{u_{i}(q)\right\}$ has been computed at the points $\left\{t_{i}\right\}, i=1-m, \cdots, J+m$, this solution is extended by shooting with the same difference formula of order $\mathcal{O}\left(h^{2 q+2}\right)$. The formulas for shooting are then obtained from (2.2) with the correction terms (4.4a) added. This yields:

$$
\begin{array}{r}
u_{i}(q)=C_{-m}^{-1}\left[f^{h}\left(t_{i+m}\right)+S_{1, q}\left[u_{i+m}(q-1)\right]-\sum_{s=-m+1}^{m} C_{s}\left(t_{i+m}, h\right) u_{s+i+m}\right], \\
i=-m, \cdots,-m-r_{q+1, \bar{q}},
\end{array}
$$

and

$$
u_{i}(q)=C_{m}^{-1}\left[f^{h}\left(t_{i-m}\right)+S_{1, q}\left[u_{i-m}(q-1)\right]-\sum_{s=-m}^{m-1} C_{s}\left(t_{i-m}, h\right) u_{s+i-m}\right],
$$

Here $\bar{q}$ is the maximum number of corrections intended (see $\S 5.1$ ).

Deferred correction formulae. Following a procedure similar to the one given in Lemma 2.15 (although much simpler), it is possible to derive centered finite difference approximations of order higher than 2 to the derivatives $\mathscr{D}^{r}(y), r=1,2, \cdots,[10]$. They have the form

$$
h^{r} D_{2 q, \theta}^{r} y_{i} \equiv \sum_{s=-\underline{m}_{r, q}}^{\bar{m}_{r, q}} w_{s}^{r, q, \theta} y_{i+s}=h^{r} \mathscr{D}^{r} y_{i}+O\left(h^{r+2 q}\right),
$$

where the weights $w_{s}^{r, q, \theta}$ are chosen so that $D_{2 q, \theta}^{r} y_{i}$ is an $\mathcal{O}\left(h^{2 q}\right)$ approximation to the $r$ th derivative of $y(t)$ at $t=t_{i} \in \pi_{h}^{\theta}$. The semilengths of the formulas $\underline{m}_{r, q}, \bar{m}_{r, q}$ are given by:

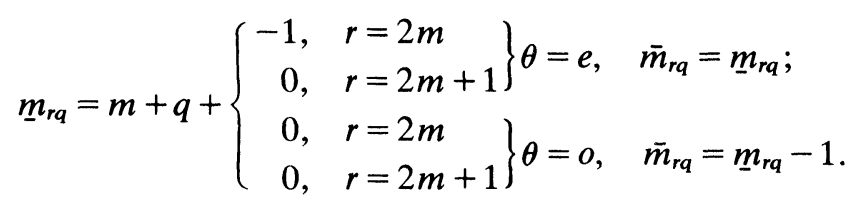

Recalling formulas (2.11), (2.13), (4.4) (for $p=1$, which we omit), and using (6.5) to approximate the derivatives to the required order we obtain

$$
S_{q}\left[u_{j}(q-1)\right]=\sum_{\nu=1}^{q} h^{2 \nu} \sum_{\mu=0}^{n} \alpha_{\nu \mu}^{\theta} A_{\mu}\left(t_{j}\right) \sum_{s=-\underline{m}_{\mu+2 \nu, \tilde{q}}}^{\bar{m}_{\mu+2 \nu, \tilde{q}}} h^{-(\mu+2 \nu)} w_{s}^{\mu+2 \nu, \tilde{q}, \theta} u_{j+s}(q-1),
$$

where $\tilde{q}=q-\nu+1$. Similarly the correction operators for the boundary conditions are

$$
\begin{aligned}
S B_{k, q}\left[u^{h}(q-1)\right]= & \sum_{\nu=1}^{q} h^{2 \nu} \sum_{\mu=0}^{n=1} \beta_{\nu \mu}^{\theta} h^{-(\mu+2 \nu)} \\
& \times\left[B_{k \mu}(a) \sum_{s=-m_{\mu+2 \nu, \tilde{q}}}^{\bar{m}_{\mu+2 \nu, \tilde{q}}} w_{s}^{\mu+2 \nu, \tilde{q}, \theta} \mu_{s}(q+1)\right. \\
& \left.\quad+\sum_{s=-m_{\mu+2 \nu \tilde{q}}}^{\bar{m}_{\mu+2 \nu, \tilde{q}}} w^{\mu+2 \nu, \tilde{q}, \theta} \mu_{J+s}(q-1)\right] .
\end{aligned}
$$


Reordering these summations we can obtain a format similar to (2.2), but now we have to separate the cases $\theta=e, o$. In an obvious notation (6.7a) becomes

$$
\begin{array}{r}
S_{q j}^{e}=\sum_{s=-(m+q)}^{m+q}\left\{\sum _ { \nu = 1 } ^ { q } \sum _ { \mu = \operatorname { m a x } ( 0 , | s | - q ) } ^ { m } \left[\left(w_{s}^{2(\mu+\nu), \tilde{q}, e} \alpha_{\nu, 2 \mu}^{e}\right) A_{2 \mu}\left(t_{j}\right) h^{-2 \mu}\right.\right. \\
\left.\left.+\left(w_{s}^{2(\mu+\nu)-1, \tilde{q}, e} \alpha_{\nu, 2 \mu-1}^{e}\right) A_{2 \mu-1}\left(t_{j}\right) h^{-(2 \mu-1)}\right]\right\} u_{j+s}(q-1) \\
q=1, \cdots, \bar{q} ; j=j_{q}, \cdots, J_{q},
\end{array}
$$

where $j_{q}=-r_{q, \bar{q}}+1, J_{q}=J+r_{q, \bar{q}}$, and $\bar{q}$ is the maximum number of corrections intended. The $r_{q, \bar{q}}$ are defined in (5.3). Similarly, if we put $\mu_{0}=\max \left(0,\left|s+\frac{1}{2}\right|-\left(q+\frac{1}{2}\right)\right)$ and for $x=a, b$ we define

$$
\begin{aligned}
\Gamma_{s}(x) \equiv \sum_{\mu=\mu_{0}}^{m-1}[ & \sum_{\nu=1}^{q}\left(w_{s}^{2(\mu+\nu), \tilde{q}, e} \beta_{\nu, 2 \mu}^{e}\right) B_{k, 2 \mu}(x) h^{-2 \mu} \\
& \left.+\sum_{\nu=1}^{q}\left(w_{s}^{2(\mu+\nu)+1, \tilde{q}, e} \beta_{\nu, 2 \mu+1}^{e}\right) B_{k, 2 \mu+1}(x) h^{-(2 \mu+1)}\right],
\end{aligned}
$$

then $(6.7 b)$ becomes:

$$
S B_{k, q}^{e}=\sum_{s=-(m+q)}^{m+q-1}\left[\Gamma_{s}(a) u_{s}(q-1)+\Gamma_{s}(b) u_{J+s}(q-1)\right],
$$

In the odd case we obtain,

$$
\begin{aligned}
S_{q j}^{o}=\sum_{s=-(m+q)}^{m+q}\left\{\sum_{\mu=\mu_{0}}^{m}\right. & {\left[\sum_{\nu=1}^{q}\left(w_{s}^{2(\mu+\nu), \tilde{q}, o} \alpha_{\nu, 2 \mu}^{o}\right) A_{2 \mu}\left(t_{j}\right) h^{-2 \mu}\right.} \\
& \left.\left.+\sum_{\nu=1}^{q}\left(w_{s}^{2(\mu+\nu)+1, \tilde{q}, o} \alpha_{\nu, 2 \mu+1}^{o}\right) A_{2 \mu+1}\left(t_{j}\right) h^{-(2 \mu+1)}\right]\right\} u_{j+s}(q-1) .
\end{aligned}
$$

For the boundary conditions we get,

$$
\begin{array}{r}
S B_{k, q}^{o}=\sum_{s=-(m+q+1)}^{m+q} \sum_{\nu=1}^{q}\left\{\sum_{\mu=\mu_{0}}^{m} h^{-2 \mu} \beta_{\nu, 2 \mu}^{o} w_{s}^{2(\mu+\nu), \tilde{q}, o}\left(B_{k, 2 \mu}(a) u_{s}+B_{k, 2 \mu}(b) u_{J+s}\right)\right. \\
+\sum_{\mu=\mu_{0}}^{m-1} h^{-(2 \mu+1)} \beta_{\nu, 2 \mu+1}^{o} w_{s-1}^{2(\mu+\nu)+1, \tilde{q}, o}\left(B_{k, 2 \mu+1}(a) u_{s-1}(q-1)\right. \\
\left.\left.+B_{k, 2 \mu+1}(b) u_{J+s-1}(q-1)\right)\right\} .
\end{array}
$$

7. High- vs. low-order systems; an example. It is of interest to compare the operational count or equivalently the computer time used to solve a given problem which can be formulated both as a high order system or as a lower order system. Of course all problems can be written as first order systems. But should we bother to do this? We shall make some simple theoretical estimates and then show how well they apply in a specific example (the Orr-Sommerfeld equation) formulated in three different ways.

The most time consuming part of the solution procedure is in general (i.e. assuming easily evaluated coefficients in the differential equations) that concerned with solving the linear systems (6.2) with coefficients of the form (6.3). 
We have used a band Gaussian elimination code with partial pivoting for the solution of these linear equations. The number of operations for solving one system with such an algorithm is essentially

$$
\text { Ops }=2 N \beta^{2} \text {. }
$$

Here $N$ is the algebraic order of the system and $\beta$ is the half bandwidth given in (6.4). This may not be the most efficient way of solving such sets of equations. However it is fairly good and we have used it in our estimates and in our test calculations.

Let us assume that $n$, the order of the system, can be factored as $n=r \times s$ with $r$, say, even. We estimate the number of operations required to solve the problem as a system of $d$ equations of order $n$, as a system of $d \times s$ equations or order $r$ and finally as a system of $d \times n$ equations of the first order. For simplicity we assume that the number of initial conditions $p=n d / 2$, i.e. the same number of boundary constraints at each endpoint. In this case, it is easy to check that the bandwidth (6.4) is $\beta=d n-1$. See Table 3 . Let $J$ again be the number of intervals. Then the number of linear equations [i.e. the algebraic order of (6.2)] is: $N=(J+r) d s$. In Table 3 we give the leading terms in the number of arithmetic operations needed for various combinations of these parameters. We can then answer the question: Is it faster to solve a given problem as a high order differential system or as a larger but lower order differential system?

TABLE 3

Operational counts to solve a system of $d$ equations of order $n=r$ formulated in three different ways.

\begin{tabular}{ccccc}
\hline $\begin{array}{c}\text { Order of } \\
\text { diff. eqs. }\end{array}$ & $\begin{array}{c}\text { No. of diff. } \\
\text { eqs. }\end{array}$ & $\begin{array}{c}\text { No. of linear } \\
\text { eqs. }\end{array}$ & $\beta$ & Ops. \\
\hline$n=r \times s$ & $\begin{array}{c}d \times s \\
r\end{array}$ & $\begin{array}{c}(J+n) \times d \\
(J+r) \times d \times s \\
J \times d \times n\end{array}$ & $\begin{array}{c}n \times d \\
n \times d \\
\frac{3}{2} d n\end{array}$ & $\begin{array}{c}2(J+n) n^{2} d^{3} \\
2(J+r) n^{2} d^{3} s \\
\frac{9}{2} J d^{3} n^{3}\end{array}$ \\
\hline$\hat{1}$ & $d \times n$ & $J \times d \times n$ & $J d^{3} n^{3}$ \\
\hline
\end{tabular}

The number of operations, Ops $\hat{1}$, for a first order system is based on using a tridiagonal block solver with alternate pivoting, as the one described in [6], while $\mathrm{Ops}_{1}$ corresponds to the band solver yielding (7.1).

The ratios between these number of operations (subindicated by the order) are

$$
\frac{\mathrm{Ops}_{r}}{\mathrm{Ops}_{n}}=s, \quad \frac{\mathrm{Ops}_{1}}{\mathrm{Ops}_{n}}=\frac{9 n}{4}, \quad \frac{\mathrm{Ops}_{\hat{1}}}{\mathrm{Ops}_{n}}=\frac{n}{2} .
$$

So reducing the original system of $d$ equations of order $n$ to one with $d s$ equations of order $r$ will increase the number of operations in the linear equation solver by the factor $s$; in a similar fashion, reduction to a first order system will be more costly for $n>2$, even if the very efficient linear equations solver of [6] is used.

Since solution of the linear equations is the main part of the computation that will be affected by these reductions we conclude that it is faster to keep the system as a high order one, especially if $n>2$. Recall however that we have not accounted for the ease in using a nonuniform net for first order systems. So they are by no means ruled out as efficient schemes for obtaining accurate solutions, see [13], [21].

To check the above theoretical estimates we consider the Orr-Sommerfeld equations in the case of plane Poiseuille flow between stationary parallel plates located at 
$x= \pm 1$. Decomposed into real and imaginary parts this yields the system of two $(d=2)$ fourth order $(n=4)$ linear differential equations:

$$
\begin{aligned}
& y_{1}^{(\mathrm{IV})}-2 \alpha^{2} y_{1}^{(\mathrm{II})}+\alpha^{4} y_{1}+\alpha R\left[(U-c)\left(y_{2}^{(\mathrm{II})}-\alpha^{2} y_{2}\right)+2 y_{2}\right]=0, \\
& y_{2}^{(\mathrm{IV})}-2 \alpha^{2} y_{2}^{(\mathrm{II})}+\alpha^{4} y_{2}-\alpha R\left[(U-c)\left(y_{1}^{(\mathrm{II})}-\alpha^{2} y_{1}\right)+2 y_{1}\right]=0 .
\end{aligned}
$$

Here $U(x)=1-x^{2}$ is the given velocity field and $\alpha, c, R$ are for the present study, real constants. Although the Orr-Sommerfeld equation is usually posed with homogeneous boundary conditions to yield an eigenvalue problem, we take here the inhomogeneous boundary conditions:

$$
\begin{aligned}
& y_{1}(-1)=y_{1}^{(\mathrm{I})}(-1)=y_{2}^{(\mathrm{I})}(-1)=0, \\
& y_{1}(0)=1, \quad y_{1}^{(\mathrm{I})}(0)=y_{2}^{(\mathrm{I})}(0)=y_{1}^{(\mathrm{III})}(0)=y_{2}^{(\mathrm{III})}(0)=0 .
\end{aligned}
$$

In this way (7.3)-(7.4) is a two point boundary value problem whose solution is but one step in the iterative search for eigensolutions of a basic stability problem in fluid mechanics. We do not give more details here but it should be clear that efficient methods for solving the unit problem above are of great value.

We have solved the problem posed in (7.3)-(7.4) using the implementations described above with the given formulation (i.e. two fourth order equations), as a system of four second order equations (by introducing $z_{1} \equiv y_{1}^{(\mathrm{II})}$ and $z_{2} \equiv y_{2}^{(\mathrm{II})}$ ) and as a system of eight first order equations. This final formulation was implemented with both a band elimination and block-tridiagonal solver. In Table 4 we give some observed computation times (on an IBM 370/158 computer) for these codes using the constants: $\alpha=0.991684, c=0.259978, R=5846$ and with $J=100$ points.

TABLE 4

Times to solve the Orr-Sommerfeld equation formulated in three different ways.

\begin{tabular}{lcccccc}
\hline \multicolumn{1}{c}{ Soln. method } & $\begin{array}{c}\text { Order of } \\
\text { O.D.E.'s }\end{array}$ & $\begin{array}{c}\text { No. of } \\
\text { O.D.E.'s }\end{array}$ & $\begin{array}{c}\text { Setup time } \\
(\mathrm{ms})\end{array}$ & $\begin{array}{c}\text { Soln. time } \\
(\mathrm{ms})\end{array}$ & $\begin{array}{c}\text { Observed } \\
\text { ratios }\end{array}$ & $\begin{array}{c}\text { Theoret. } \\
\text { ratios }\end{array}$ \\
\hline Even band elim. & 4 & 2 & 841 & 1930 & 1 & 1 \\
Even band elim. & 2 & 4 & 1043 & 3757 & 1.95 & 2 \\
Odd band elim. & 1 & 8 & 1547 & 16391 & 8.5 & 9 \\
Block tridiag. & 1 & 8 & 1075 & 4196 & 2.1 & 2 \\
\hline
\end{tabular}

\section{REFERENCES}

[1] L. Collatz, The Numerical Treatment of Differential Equations, 3rd ed., Springer-Verlag, Berlin, 1960.

[2] J. W. Daniel, V. Pereyra And L. L. Schumaker, Iterated deferred corrections for initial value problems, Acta Ci. Venezolana, 19 (1968), pp. 128-135.

[3] L. Fox, Numerical Solution of Two Point Boundary Value Problems, Clarendon Press, Oxford, 1957.

[4] H. B. Keller, Numerical Methods for Two Point Boundary Value Problems, Ginn-Blaisdell, Waltham, MA, 1968.

[5] - Accurate difference methods for linear ordinary differential systems subject to linear constraints, this Journal, 6 (1969), pp. 8-30.

[6] - Accurate difference methods for nonlinear two-point boundary value problems, this Journal, 11 (1974), pp. 305-320.

[7] - Approximation methods for nonlinear problems with applications to two point boundary value problems, Math. Comput., 29 (1975), pp. 464-474. 
[8] - Numerical solution of boundary value problems for ordinary differential equations: Survey and some recent result on difference methods, Numerical Solution of Boundary Value Problems for Ordinary Differential Equations, A. K. Aziz, ed., Academic Press, New York, 1975.

[9] - Numerical Solution of Two Point Boundary Value Problems, CBMS Regional Conference Series in Applied Math., vol. 24; Society for Industrial and Applied Mathematics, Philadelphia, PA, 1976.

[10] H. B. Keller AND V. Pereyra, Symbolic generation of finite difference formulae, Math. Comput., to appear.

[11] H. B. Keller AND A. B. White, JR., Difference methods for boundary value problems in ordinary differential equations, this Journal, 12 (1975), pp. 791-802.

[12] H.-O. KREISS, Difference approximations for ordinary differential equations, Math. Comput., 26 (1972), pp. 605-624.

[13] M. LENTINI AND V. PEREYRA, A variable order finite difference method for nonlinear multipoint boundary value problems, Ibid., 28 (1974), pp. 981-1004.

[14] V. PEREYRA, Iterated deferred corrections for nonlinear operator equations, Numer. Math., 10 (1967), pp. 316-323.

[15] — Iterated deferred corrections for nonlinear boundary value problems, Ibid., 11 (1968), pp. 111-125.

[16] - Highly accurate numerical solution of casi-linear elliptic boundary value problems in $n$ dimensions, Math. Comput., 24 (1970), pp. 771-783.

[17] V. PEREYRA, High order finite difference solution of differential equations, Stanford Univ. Comp. Sc. Dept., STAN-CS-73-348, 1973.

[18] V. PEREyRA, W. Proskurowski AND O. WidLund, High order fast Laplace solvers for the Dirichlet problem on general regions, Math. Comput., 31 (1977), pp. 1-16.

[19] H. J. STETTER, Asymtotic expansions for the error of discretization algorithms for non-linear functional equations, Numer. Math., 7 (1965), pp. 18-31.

[20] A. B. White, JR., Numerical Solution of Two Point Boundary Value Problems, Ph.D. thesis, California Inst. of Tech., Pasadena, CA, 1974.

[21] M. LENTINI AND V. PEREYRA, An adaptive finite difference solver for nonlinear two-point boundary value problems with mild boundary layers, this Journal, 14 (1977), pp. 91-111.

[22] J. H. CERUTTI, Collocation for systems of ordinary differential equations, Comp. Sci. Tech. Rep. No. 230, U. Wisconsin, Madison, 1974. 